\title{
Spermatogenesis and Nucleolar Activity in Triatoma klugi (Triatomine, Heteroptera)
}

\author{
Laiana Cristina da Costa, Maria Tercília Vilela de Azeredo-Oliveira and Ester Tartarotti \\ Departamento de Biologia, Instituto de Biociências, Letras e Ciências Exatas, \\ Universidade Estadual Paulista “Julio de Mesquita Filho”, São José do Rio Preto, SP, Brazil.
}

\begin{abstract}
Triatoma klugi is a Chagas disease vector in the Rio Grande do Sul State. Triatominae chromosomes are holocentric and sex chromosomes segregation is post-reductional. In this paper we describe the karyotype of male $T$. klugiand a meiotic analysis including the nucleolar behavior during spermatogenesis. Testis cells were analyzed after lactoacetic orcein and silver nitrate staining. Two autosomes and the heterochromosomes presented nucleolar activity (Ag-NORs) during diplotene-diakinesis. The analysis of metaphase I and II revealed a karyotype with $2 n=20+X Y$. In metaphase I a prominent nucleolar mass was observed in the cell periphery and small silver grains were detected in metaphase II. During anaphase, the chromosomes segregated in parallel and a typical holocentric late migration behavior was observed. The restoration of the nucleolus was an important feature in this phase. During telophase nucleolar masses persisted and in early spermiogenesis the spermatids presented a small peripheral mass until elongation. The present study is a contribution to the study of chromatin behavior and nucleolar persistence in meiosis.
\end{abstract}

Key words: Triatoma klugi, Triatomine, cytogenetics, nucleolus, karyotype, meiosis.

Received: August 27, 2007; Accepted: January 16, 2008.

\section{Introduction}

The hematophagous insects belonging to the Triatominae subfamily are best known as triatomines. These arthropods are vectors of the Chagas disease etiological agent, the protozoan Trypanosoma cruzi (T. cruzi). The disease is endemic in South America with pronounced relevance in heart illnesses. It is considered that T. cruzi infects about 11 million people in endemic regions (Dias et al., 2002). Chagas disease represents the third most common parasitic affection, after malaria and schistosomiasis (Guzmán-Bracho, 2001).

The Triatominae subfamily includes 137 insect species (Galvão et al., 2003) divided into six tribes: Alberproseniini, Bolboderini, Cavernicolini, Linshcosteini, Rhodniini, and Triatomini. Eighteen triatomine genera have been described: Alberprosenia, Belminus, Bolbodera, Microtriatoma, Parabelminus, Cavernicolai, Linshcosteus, Psammolestes, Rhodnius, Dipetalogaster, Eratyrus, Hermanlentia, Meccus, Mepraia, Nesotriatoma, Paratriatoma, Panstrongylus, and Triatoma (Galvão et al., 2003).

Send correspondence to Ester Tartarotti. Departamento de Biologia, Instituto de Biociências, Letras e Ciências Exatas, Universidade Estadual Paulista "Julio de Mesquita Filho", Rua Cristóvão Colombo 2265, Jardim Nazareth, 15054-000 São José do Rio Preto, SP, Brazil. E-mail: tartarot@ ibilce.unesp.br.
Triatomines are homogeneous in relation to their hematophagous behavior and all species are potential vectors of Chagas disease (Tartarotti et al., 2004). Triatoma klugi (T. klugi) is an important vector in sylvan environments and is distributed throughout the state of Rio Grande do Sul. This species is part of the "oliverai complex", which also includes T. matogrossensis, T. williami, T. guazu and T. jurbergi (Carcavallo et al., 2001). Triatoma klugi specimens susceptible to different lineages of Trypanosoma cruzi showed an infection similar to that observed in Triatoma infestans (T. infestans) (Joukoski et al., 2000). Under laboratory conditions this species presented an infection rate for the $T$. cruzi lineages $60 \%$ higher than previously observed (Emmanuelle-Machado et al., 2002).

Cytogenetic studies revealed that the typical number of chromosomes in Triatominae is $2 \mathrm{n}=22$, with 20 autosomes and two sex chromosomes (XX, XY) (Ueshima, 1979). Most species have 20 autosomes, except for Triatoma rubrofasciata (22A), Panstrongylus megistus and $T$. nitida (18A) (Dujardin et al., 2000). Fifty species have had their karyotypes described and 30 of them were shown to possess 2n = 20A $+\mathrm{XY}$ (Panzera et al., 1998; Morielle and Azeredo-Oliveira, 2004). The variation in the number of chromosomes was mainly due to the presence of different sex determining systems. The subfamily displayed a frag- 
mentation of the males $X$ chromosome and the following systems have been observed: $\mathrm{XY}, \mathrm{X}_{1} \mathrm{X}_{2} \mathrm{Y}$ and $\mathrm{X}_{1} \mathrm{X}_{2} \mathrm{X}_{3} \mathrm{Y}$. These three systems have been observed in the genus Triatoma (Panzera et al., 1998). A fourth sex determination mechanism, $\mathrm{X}_{1} \mathrm{X}_{2} \mathrm{Y}_{1} \mathrm{Y}_{2}$, has been recently described in Mepraia spinolai (Frías and Atria, 1998).

Triatomines have holokinetic chromosomes, which are characterized by the absence of centromere in the primary chromosome constriction (Pérez et al., 2000). These insects possess a peculiar behavior during meiosis, in which the first meiotic division is reductional for autosomes and equational for the sex chromosomes (GonzálezGarcía et al., 1996).

Several authors have studied the nucleolus organizer regions (NORs) in Heteroptera (Camacho et al., 1985; Fossey and Liebenberg, 1995; Cattani and Papeschi, 2004). In the subfamily Triatominae the nucleolus persists during meiosis (Tartarotti and Azeredo-Oliveira, 1999b; Morielle and Azeredo-Oliveira, 2004). Some studies performed in insects indicated that silver nitrate stained structures could represent kinetochore or centromere material (Suja et al., 1991; Rufas and Gosálvez, 1982). Ultrastructural analysis of Tricholepidion (Insecta) spermiogenesis evidenced a lump of diffuse granular material, the related to the centriole in early spermatids (Dallai et al., 2001). This structure resembles the nucleolar bodies present in triatomine spermatids. Triatoma klugi has been recently described by Carcavallo et al. (2001) and it is morphologically related to T. oliveirai, justifying its inclusion in the oliveirai complex (Emmanuelle-Machado et al., 2002). The purpose of this paper was to describe the karyotype of male Triatoma klugi and to analyze the chromosomes meiotic behavior and nucleolar cycle during spermatogenesis.

\section{Materials and Methods}

Specimens of Triatoma klugi (subfamily Triatominae, family Reduviidae, order Heteroptera; Carcavallo et al., 2001) were supplied by the Araraquara Special Health Service (SESA), part of the Epidemiology Department of the Public Health Faculty of University of São Paulo (USP).

Triatoma klugi is found in gaps between rocks and is distributed throughout the district of Nova Petrópolis, in the state of Rio Grande do Sul. These insects are predominantly black; with a central yellow spot on each connexivum segment (Carcavallo et al., 2001).

We analyzed testes from 20 young adults. After the seminiferous tubules were squashed, part of the material was stained with lacto-acetic orcein (De Vaio et al. 1985) and part was submitted to silver nitrate staining (Howell and Black, 1980). Measurements of the sex chromosomes were performed with the Image Tool version 3 for Windows (UTHSCSA, 1995/2002) in ten metaphase I cells. The photomicrographs were taken in Zeiss-Jenaval photomicroscope.

\section{Results}

\section{Karyotype and meiosis of Triatoma klugi}

Triatoma klugi males (Figure 1a) presented a karyotype with $2 \mathrm{n}=22$, consisting of 20 autosomes and a $\mathrm{XY}$ pair (Figure 1b). The Y chromosome $(2,21 \pm 0,12 \mu \mathrm{m})$ was larger than the $X(1,85 \pm 0,16 \mu \mathrm{m})$. The polyploid nuclei of nutritious cells from the seminiferous tubules walls presented on average five positive heteropycnotic corpuscles (Figure 2a). In spermatogonial interphase cells, six positive heteropycnotic corpuscles were observed (Figure 2b). In the diffuse stage of prophase I, the nuclei had one or two positive heteropycnotic corpuscles, probably formed by sex chromosomes (Figure 2c-d), and were more compacted during the diffuse phase (Figure 2 e-f). During diplotenediakinesis autosome bivalents presented chiasmata and the sex chromosomes were highly condensed (Figure $2 \mathrm{~g}$ ). At metaphase I the ten autosome bivalents and the sex chromosomes were arranged on the metaphase plate (Figure 3a) and usually disposed in a ring configuration (Figure 3b). In metaphase II, ten autosomes and one sex chromosome were present (Figure 3c-d). During anaphase II, late migrating chromosomes were seen (Figure 3e-f), which were sometimes also observed later at telophase II (Figure 3g). Positive heteropycnotic corpuscles were observed in early spermatids, but not in (Figure 3h) cells at more advanced differentiation stages (Figure 3i).

\section{Silver nitrate staining}

After silver nitrate staining the polyploid nuclei of nutritious cells from the seminiferous tubules walls presented a central nucleolus (Figure 4a). Chromosomes joined by chromatin filaments were observed at spermatogonial metaphases (Figure 4b), while a central/lateral nucleolus and small nucleolar bodies could be seen in spermatocytes (Figure 4c). Dispersed nucleolar masses were present at the beginning of the diffuse stage (Figure 4d) and during diplotene-diakinesis the sex chromosomes sometimes presented an active NOR (Figures $4 \mathrm{e}-\mathrm{g}$ ). At these same stages NORs were detected on two autosome bivalents (Figure $4 \mathrm{~g}$ ) or, alternatively, a prominent NOR was visible in one autosome bivalent (Figure 5a); in both situa-

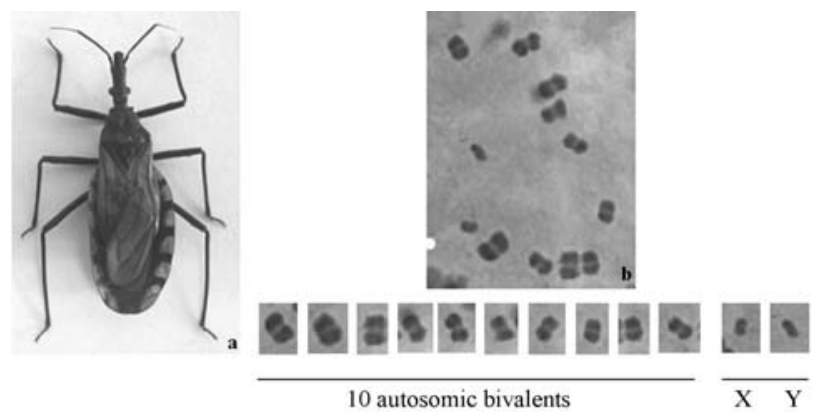

Figure 1 - a) Triatoma klugi male; b) Metaphase I and karyotype with ten bivalent autosomes and two sex chromosomes (XY). 

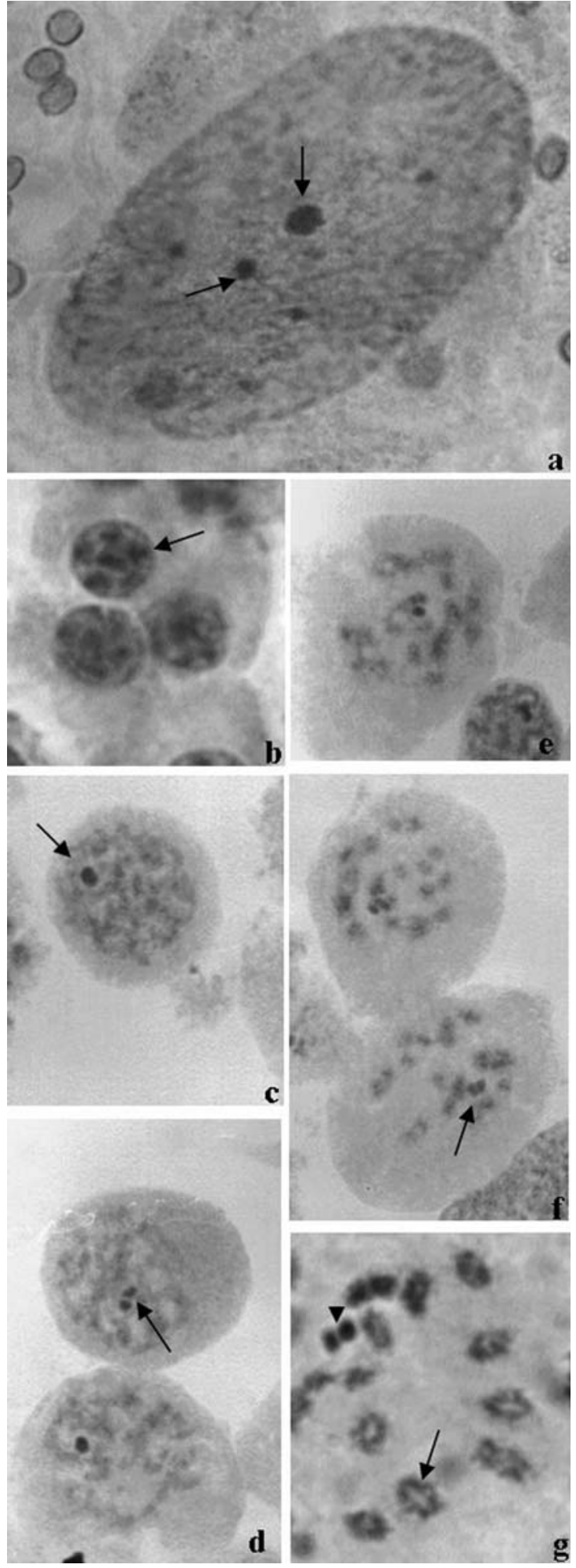

Figure 2 - Testicular tubules of Triatoma klugi after lacto-acetic orcein staining. (a) Polyploid nuclei with on average five heteropycnotic corpuscles, arrows indicate corpuscles; (b) Spermatogonial interphase cells with six pycnotic corpuscles, the arrow indicates the corpuscles; (c-d) Nuclei at the beginning of prophase I (diffuse stage) presenting one or two condensed corpuscles formed by the sex chromosomes, arrows indicate corpuscles; (e-f) Nuclei in early prophase I, sexual corpuscle is indicated by arrow; (g) Nucleus in diplotene-diakinesis, arrow indicates bivalent autosomes and the arrowhead points to the pseudobivalent sex chromosomes.
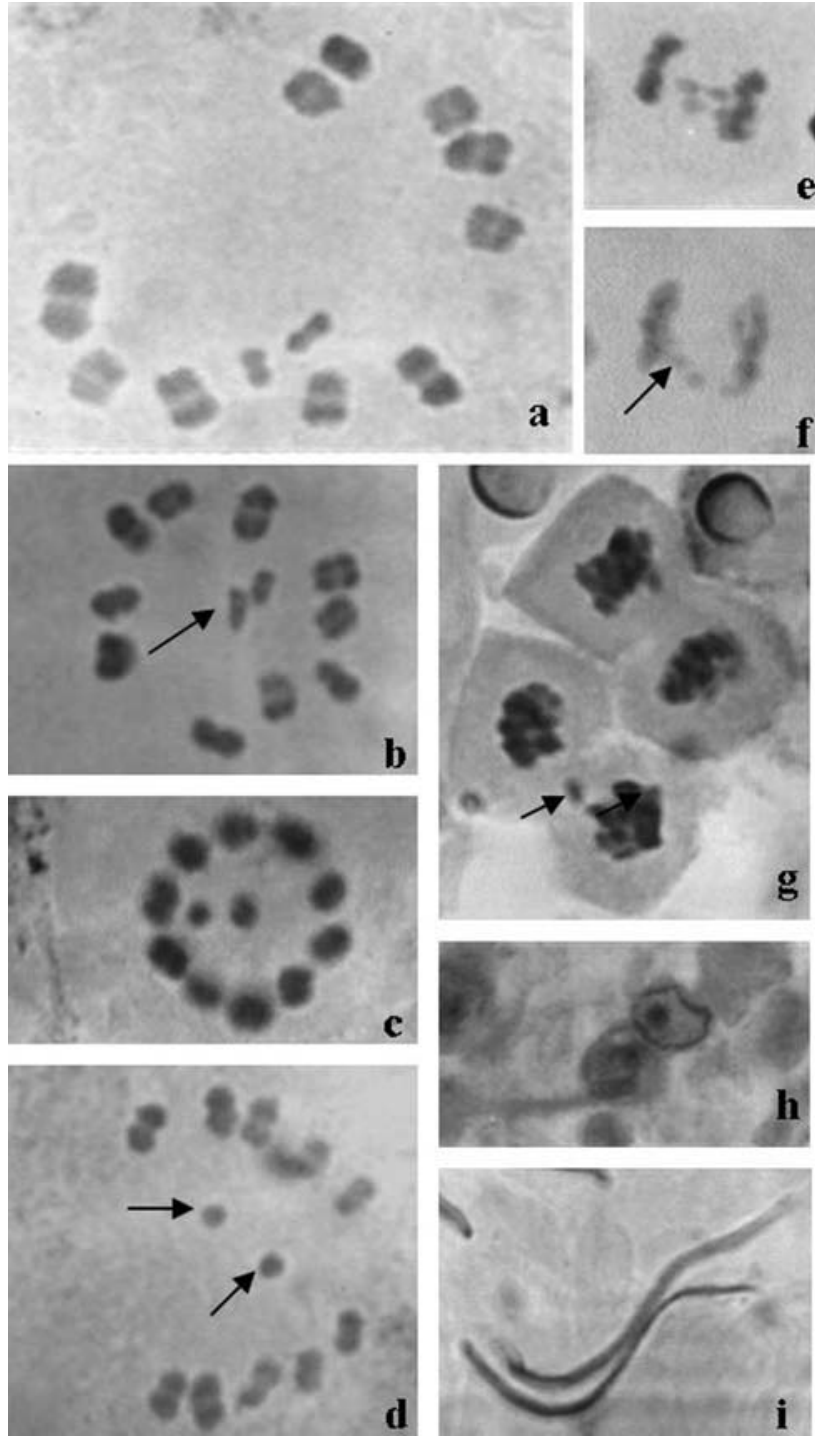

Figure 3 - Testicular tubules of Triatoma klugi after lacto-acetic orcein staining: (a-b) Metaphase I with ten bivalent autosomes and two sex chromosomes indicated by the arrow; (c-d) Metaphase II with equational division of the sex chromosomes shown by arrows. Note that in (c) the chromosomes are arranged in a ring; (e-f) Anaphase with late chromosomes migration, the arrow indicates parallel chromosome migration; (g) Telophase II with four gametic cells, the arrow indicates late migrating chromosomes; (h) Spermatids with heteropycnotic corpuscles in early spermiogenesis, (i) Sperm cells at the advanced differentiation stage.

tions the sex chromosomes presented inactive NORs (Figure $4 \mathrm{~g}$ and $5 \mathrm{a}$ ). A large peripheral nucleolar mass was observed during metaphase I (Figure 5b) and small silver stained corpuscles were common during metaphase II (Figure $5 \mathrm{c}$ ). In this phase, at least one autosome presented an active NOR (Figure 5d). At anaphase, the chromosomes segregated to the poles in a parallel configuration and were late migrating, as it is typical for holokinetic chromosomes (Figure 5e). In this stage a prominent nucleolar mass reappeared (Figure 5f) and at telophase nucleolar remnants could be detected (Figure 5g). Early spermatids also had a small peripheral nucleolar mass (Figure $5 \mathrm{~h}$ ) and during the 

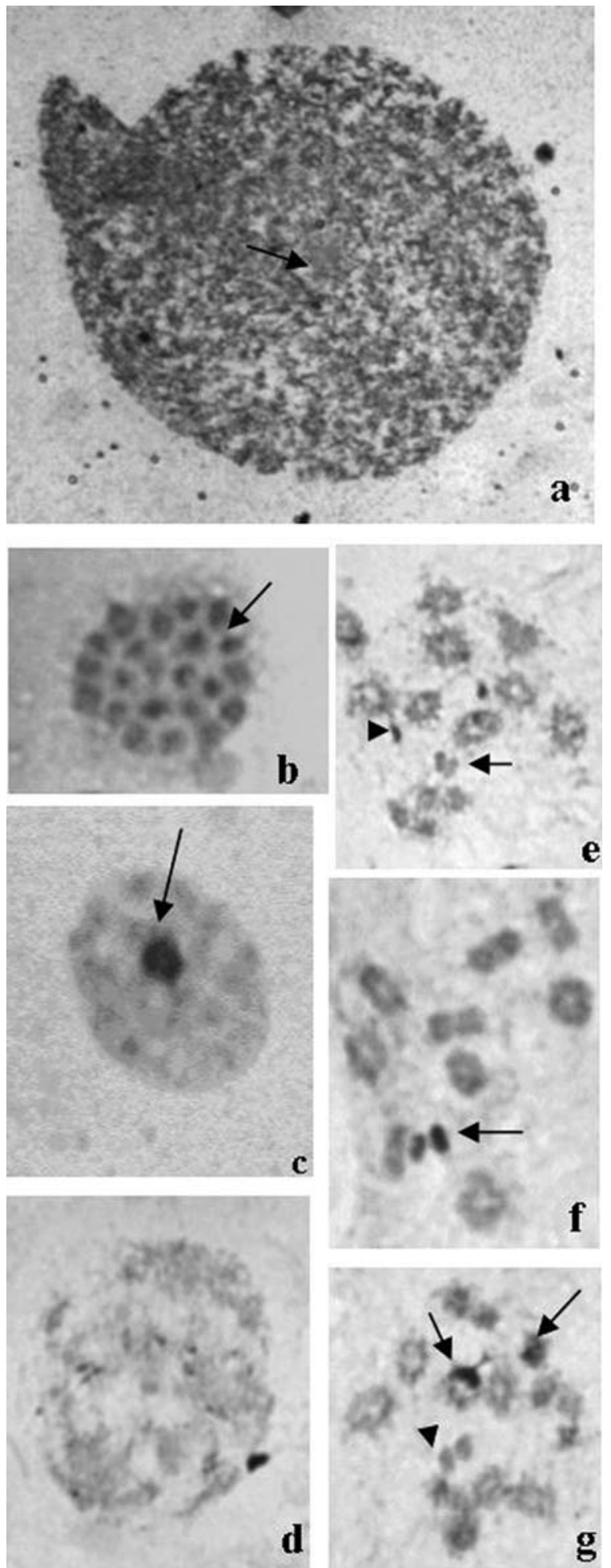

f

Figure 4 - Silver-impregnated testis tubules of Triatoma klugi: (a) Polyploid nuclei of nutritive cells of tubule wall with a silver stained nucleolus indicated by arrow; (b) Spermatogonial metaphase with chromatin fibers joining the chromosomes indicated by arrow; (c) Spermatocytes, the arrow indicates a silver stained nucleolus; (d) Nucleus in prophase I with a peripheral nucleolar corpuscle; (e) Diplotene, the arrow indicates the sex pseudobivalent. Note the nucleolar corpuscle shown by the arrowhead; (f) Diakinesis, arrow shows the silver stained sex pseudobivalent; (g) Nucleus in diplotene-diakinesis, silver stained autosomes are indicated by arrows and arrowhead indicates unimpregnated sex chromosomes. elongation stage, spermatids still presented a small silver stained mass (Figure $5 \mathrm{i}$ ). The masses seen in Figures $5 \mathrm{~h}$ and 5 i probably represent the centriole adjunt already observed in ultrastructural studies in insects (Dallai et al. 2001).

\section{Discussion}

Heteroptera have been very important for cytogenetic studies because they possess holocentric chromosomes, late chromosome migration in anaphase and a diffuse stage that lasts from prophase I until early diplotene (Ueshima, 1979; Hughes-Schrader and Schrader, 1961). The typical autosome number (A) in triatomines is 20 (Panzera et al., 1996) and male Triatoma klugi had 20 autosomes and two sex chromosomes $(20 \mathrm{~A}+\mathrm{XY})$. This diploid number is the same found in $T$. brasiliensis, $T$. infestans, $T$. guasayana, $T$. lecticularia, T. matogrossensis, T. pseudomaculata, T. rubrovaria, T. sordida, T. circummaculata, T. delpontei, $T$. dimidiata, T. maculata, T. melanosoma, T. pallidipennis, T. patagonica and T.platensis (Panzera et al. 1996; Panzera et al., 1998). Additionally, in the genus Triatoma the Y chromosome is larger than the X (Pérez et al. 1997; Pérez et al. 2000; Pérez et al., 2005), as seen in T. klugi. This karyotypical similarity indicates evolutionary conservation in Triatoma.

Meiosis in triatomines characteristically present positive heteropycnotic blocks at prophase I (Panzera et al. 1995). In T. klugi, the $\mathrm{X}$ and $\mathrm{Y}$ chromosomes were associated appearing as a positive heteropycnotic pseudobivalent at this stage. These heteropycnotic elements have already been observed in other triatomine species, such as: $T$. brasiliensis, T. lecticularis, T. rubrovaria, T. sordida (Tavares and Azeredo-Oliveira, 1997), Panstrongylus megistus, P. herreri (Tartarotti and Azeredo-Oliveira, 1999a) and T. vitticeps (Severi-Aguiar et al. 2006). However, this phenomenon is not typical of Heteroptera, in which Belostoma dentatum showed bivalent autosomes in continuous condensation while both $\mathrm{X}$ chromosomes were decondensed and negatively heteropycnotic during diakinesis (Papeschi and Bidau, 1985).

In T. klugi the $\mathrm{X}$ and $\mathrm{Y}$ chromosomes were laterally paired (pseudobivalency) at diplotene-diakinesis and the sex pseudobivalent showed a terminal association during metaphases I and II (Figure 3b). This is possibly due to the presence of available extremities at both ends which are free to interact with spindle fibers. Nevertheless, a lateral association has been observed during metaphase II in $T$. infestans (Pérez et al. 2000).

The sex chromosomes presented two kinds of configuration during metaphases I and II in T. klugi: they could be positioned at the center of a ring formed by autosomes or, alternatively, could be found at the periphery of the equatorial plate, which was usually observed in linear metaphases. Such configurations are typical of triatomines and the first type predominated in $T$. platensis, $T$. infestans and $T$. delpontei (Panzera et al., 1995). Both configurations were 


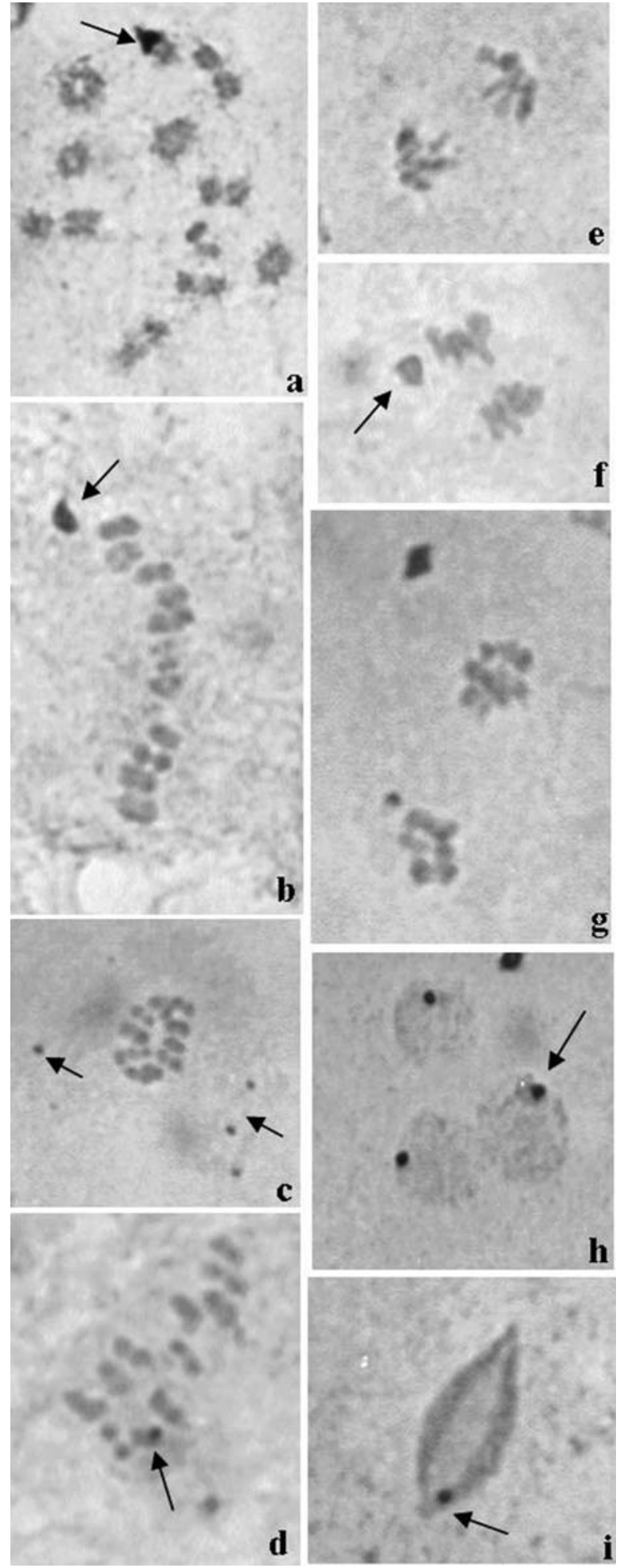

Figure 5 - Silver stained testis tubules of Triatoma klugi: (a) Diplotenediakinesis, the arrow indicates a silver stained autosome bivalent; (b) Metaphase I; the arrow indicates a large remaining nucleolar mass; (c) Metaphase II, the arrows show dispersed nucleolar masses; (d) Metaphase II with one silver stained autosome shown by arrow; (e) Anaphase I, observe the parallel migration of chromosomes; (f) Anaphase I, note the parallel chromosome disposition, the arrow indicates a prominent nucleolar mass; (g) Telophase I, note the nucleolar masses in both daughter cells; (h) Spermatids in early spermiogenesis with peripheric nucleolar mass shown by arrow; (i) Spermatids in the elongation stage with nucleolus shown by arrow. observed in Rhodnius domesticus (Morielle and AzeredoOliveira, 2004), Panstrongylus megistus and P. herreri (Tartarotti and Azeredo-Oliveira, 1999a).

Late chromosome migration was observed during meiotic anaphase in T. klugi, a phenomenon also described in Panstrongylus herreri (Tartarotti and Azeredo-Oliveira, 1999a), T. brasiliensis, T. delpontei, T. lecticularia, T. rubrovaria and T. sordida (Tavares and Azeredo-Oliveira, 1997). Late migrating chromosomes can appear either spontaneously or as a consequence of radiation in somatic or germinative cells (Hughes-Schrader and Schrader, 1961).

In T. klugi an interaction between microtubules and chromosome ends was specifically seen at anaphase I (Figure $1 \mathrm{f}-\mathrm{g}$ ). The kinetic activity was located at one extremity of the chromatids because the migration is parallel to the spindle axis. This phenomenon was also detected in $T$. infestans suggesting that the interaction of microtubules during meiosis possesses at least two steps: first, an initial holocentric interaction which determines the stabilization of chromosomes; and second, a restricted interaction towards the end that involves segregation (Pérez et al., 2000).

The restriction of kinetic activity to chromosome ends is a general characteristic of holocentric chromosomes. It has been suggested that these characteristics can facilitate the meiotic processes, such as crossing over, chiasmata terminalization and bivalent orientation on the metaphase plate (Pimpinelli and Goday, 1989).

In T. klugi the chromosomes behave as bivalents in metaphase I even after chiasmata terminalization. In Heteroptera bivalents usually display a single chiasma in any chromosome region and its presence persists beyond metaphase I (Pérez et al., 1997). The sex chromosomes of T. klugi were univalent and pseudobivalent at metaphase I and were predominantly univalent at metaphase II. The sex chromosomes appeared as univalents at metaphase I of the Antiteuchus species of heteropterans, while in metaphase II they were pseudobivalents (Lanzone and Souza, 2006). Nevertheless, a pseudobivalent XY was observed in both the first and second meiotic divisions in the Heteroptera Graphosoma italicum (González-García et al. 1996).

Silver staining revealed impregnated bodies in the polyploid nuclei of T. klugi. Nucleolar bodies have also been observed in testis polyploid nuclei of Panstrongylus megistus and P. Herreri (Tartarotti and Azeredo-Oliveira, 1999b). The increase in nucleolar bodies in polyploid nuclei may be related to increased rRNA synthesis (Tavares and Azeredo-Oliveira, 1997).

During meiotic prophase and metaphases I and II of T. klugi, an alternation of ribosomal genes activity between autosomes and sex chromosomes was observed. The same phenomenon has already been described $P$. megistus and $P$. herreri (Tartarotti and Azeredo-Oliveira, 1999b) and indicates that not all ribosomal genes are necessarily active. The presence of NORs in heterochromosomes is a common 
phenomenon in insects and several Drosophila species, for instance, have ribosomal genes located on their sex chromosomes (Bicudo, 1984).

Dispersed nucleolar bodies were observed at metaphase II in T. klugi and a large nucleolar mass was also observed during metaphase I, anaphase I and telophase I. This confirms the occurrence of "nucleolar material persistence" as suggested by Tartarotti and Azeredo-Oliveira (1999b) and provides evidence that the nucleolus does not completely disappear during meiosis in triatomines, but is reorganized from pre-existing nucleolar elements.

Nevertheless, some authors suggest that silver nitrate impregnation may also reveal other chromosome structures. Silver staining of Schistocerva gregaria (Orthoptera) early spermatids apparently revealed kinetochore or centromere material, while silver stained structures in elongated spermatids were attributed to a particular kind of condensed chromatin. Another study performed during meiosis in Orthopera led to the conclusion that silver staining reveals three distinct chromosome structures: (i) active nucleolar organizer regions (ii) kinetochores and (iii) cores that run along each chromatid except at their distal ends (Suja et al., 1991).

In T. klugi, silver staining observed in early spermatids disappears during elongation. Post-meiotic NORs reactivation has already been observed in mammalian cells submitted to silver staining (Hofgartner et al., 1979). It is possible that the conservation of post-meiotic NORs reactivation in vertebrates and invertebrates indicates the need for post-meiotic RNA synthesis related to differentiation. Alternatively, the silver stained structures seen in spermatids may represent he centriole adjunt observed in ultrastructural studies of spermiogenesis. An electron microscope analysis of Tricholepidion (insecta) showed that, after meiosis is completed, the spermatid has only a single centriole located close to the nucleus. Also connected to the centriole is a lump of diffuse granular material, named the centriole adjunt. The terms "centriole adjunt" or "postnuclear body" have been given to aggregates of granular masses, which initially accumulate around the centriole and that are believed to differentiate later into various sperm components (Dallai et al., 2001).

This study presents the description of the karyotype of male Triatoma klugi and contributes to a better understanding of chromatin and nucleolar behavior during meiosis.

\section{Acknowledgments}

The authors are grateful to Dr. José M. Soares Barata, Director of the Insectarium (Araraquara, SP), Epidemiology Department, Public Health Faculty (São Paulo, SP), and to the technicians of the Insectarium Mr. João Luis Molina Gil and Mr. João Mauricio Nóbrega da Silva Filho for providing the insects. We also thank Elza Mitiko Sato for the literature review, Hederson Vinicius de Souza for help in chromosome measurements, to David Roy Michael Mercer for the English revision, and to FAPESP for financial support.

\section{References}

Bicudo HEMC (1984) Variabilidade das regiões organizadoras de nucléolos nos eucariotos. Cienc Cult 440-447 (Abstract in English).

Camacho JPM, Belda J and Cabrero J (1985) Meiotic behaviour of the holocentric chromosomes of Nezara viridula (Insecta, Heteroptera) analysed by C-banding and silver impregnation. Can J Genet Cytol 27:490-497.

Carcavallo RU, Jurberg J, Lent H, Galvão C, Steindel M and Pinto CJC (2001) Nova espécie do complexo oliveirai (Nova denominação para o complexo matogrossensis) (Hemiptera, Reduviidae, Triatominae) do estado do Rio Grande do Sul, Brasil. Mem Inst Oswaldo Cruz 96:71-79.

Cattani MV and Papeschi AG (2004) Nucleolus organizing regions and semi-persistent nucleolus during meiosis in Spartocera fusca (Thunberg) (Coreidae, Heteropeta). Hereditas 140:105-111.

Dallai R, Lupetti P, Frati F, Nardi F and Afzelius BA (2001) Sperm ultrastructure and spermiogenesis in the relic species Tricholepidion gertschi Wygodzinsky (Insecta, Zygentoma). Tissue Cell 33:596-605.

De Vaio ES, Grucci B and Castagnino A (1985) Meiotic differences between three triatomine species (Hemiptera, Reduviidae). Genetica 67:185-191.

Dias JCP, Silveira AC and Schofield CJ (2002) The impact of Chagas control in Latin America: A review. Mem Inst Oswaldo Cruz 97:603-612.

Dujardin JP, Schofield CJ and Panzera F (2000) Les vecteurs de la maladie de Chagas. Recherches taxonomiques, biologiques et genetiques. Acad R Sci dóutre-Mer Bruxelles 24:1-162.

Emmanuelle-Machado P, Koerich LB, Joukoski DDB, Carvalho-Pinto CJ, Grisard EC and Steindel M (2002) Biology of Triatoma klugi Carcavallo, Jurberg, Lent and Galvão 2001 (Heteroptera, Reduviidae) under laboratory conditions: Effects of distinct blood sources and susceptibility to Trypanosoma cruzi and Trypanosoma rangeli, Brazil. Mem Inst Oswaldo Cruz 97:583-587.

Frias D and Atria J (1998) Chromosomal variation, macroevolution and possible parapatric specition in Mepria spinolai (Porter) (Hemiptera, Reduviidae). Genet Mol Biol 21:179-184.

Fossey A and Liebenberg H (1995) Meiosis and nucleolar structures in the stink bug Carlisis wahlbergi Stal (Coreidae, Heteroptera). Cytobios 81:7-15.

Galvão C, Carcavallo RU, Rocha DS and Jurberg J (2003) A checklist of the current valid species of the subfamily Triatominae Jeannel, 1919 (Hemiptera, Reduviidae) and their geographical distribution, with nomenclatural and taxonomic notes. Zootaxa 202:1-36.

González-García JM, Antonio C, Suja JA and Rufas JS (1996) Meiosis in holocentric chromosomes: Kinetic activity is randomly restricted to the chromatid ends of sex univalents in Graphosoma italicum (Heteroptera). Chromosome Res 4:124-132.

Guzmán-Bracho C (2001) Epidemiology of Chagas disease in Mexico: An update. Trends Parasitol 17:372-376. 
Hofgartner FJ, Schmid M, Krone W, Zenzes MT and Engel W (1979) Pattern of activity of nucleolus organizers during spermatogenesis in mammals as analyzed by silver-staining. Chromosoma 71:197-216.

Howell WM and Black DA (1980) Controlled silver staining of nucleolus organizer regions with protective colloidal developer: I-step method. Experientia 36:1014-1015.

Hughes-Schrader S and Schrader F (1961) The kinetochore of the Hemiptera. Chromosoma 12:327-350.

Joukoski DDB, Grisard EC and Steindel M (2000) Evaluation of the vectorial capacity of Triatoma klugi, a new triatomine species. Mem Inst Oswaldo Cruz 95(Suppl.II):333.

Lanzone C and Souza MJ (2006) Chromosome complement and meiosis in three species of the Neotropical bug genus Antiteuchus (Heteroptera, Pentatomidae, Discocephalinae). Genet Mol Biol 29:49-55.

Morielle A and Azeredo-Oliveira MTV (2004) Description of the nucleolar activity and karyotype in germinative cell lines of Rhodnius domesticus (Triatominae, Heteroptera). Caryologia 57:31-37.

Panzera F, Perez R, Panzera Y, Alvarez F, Scvortzoff E and Salvatella R (1995) Karyotype evolution in holocentric chromosomes of three related species of triatomines (Hemíptera-Reduviidae). Chromosome Res 3:143-150.

Panzera F, Pérez R, Hornos S, Panzera Y, Cestau R, Delgado V and Nicolini P (1996) Chromosome numbers in Triatominae (Hemíptera-Reduviidae): A review. Mem Inst Oswaldo Cruz 91:515-518.

Panzera F, Scvortzoff E, Pérez R, Panzera Y, Hornos S, Cestau R, Nicolini P, Delegado V, Alvarez F, Mazzella MC, et al. (1998) Cytogenetics of triatomines. In: Carcavallo RU, Galíndez IG, Jurberg J and Lent H (eds) Atlas dos Vetores da Doença de Chagas nas Américas. Editora Fiocruz, Rio de Janeiro, pp 621-664.

Papeschi AG and Bidau CJ (1985) Chromosome complement and male meiosis in four species of Belostoma Latreille (Heteroptera-Belostomatidae). Rev Bras Genet 8:249-261.

Perez R, Panzera F, Page J, Suja JA and Rufas JS (1997) Meiotic behaviour of holocentric chromosomes: Orientation and segregation of autosomes in Triatoma infestans (Heteroptera). Chromosome Res 5:47-56.

Pérez R, Rufas J, Suja J and Panzera F (2000) Meiosis in holocentric chromosomes: Orientation and segregation of in autosome and sex chromosomes in Triatoma infestans (Heteroptera). Chromosome Res 8:17-25.

Pérez R, Rufas J, Suja J and Panzera F (2005) Cytogenetic analysis of experimental hybrids in species of Triatominae (Hemiptera-Reduviidae). Genetica 125:261-270.

Pimpinelli S and Goday C (1989) Centromere organization in meiotic chromosomes of Paracaris univalents. Chromosoma 98:160-166.

Rufas JS and Gosálvez F (1982) Development of silver stained structures during spermatogenesis of Schistocerca gregaria (FORSK) (Orthoptera, Acrididae). Caryologia 35:261-267.

Severi-Aguiar GDC, Lourenço LB, Bicudo HEMC and AzeredoOliveira MTV (2006) Meiosis aspects and nucleolar activity in Triatoma vitticeps (Triatominae, Heteroptera). Genetica 126:141-151.

Suja JA, De La Torre J, Gimenéz-Abián JF, Garcia de la Vega C and Rufas JS (1991) Meiotic chromosome structure. Kinetochores and chromatid cores in standard and B chromosomes of Arcyptera fusca (Orthoptera) revealed by silver staining. Genome 34:19-27.

Tartarotti E and Azeredo-Oliveira MTV (1999a) Meiosis patterns of holocentric chromosomes in Triatomines genus Panstrongylus. Cytologia 64:235-240.

Tartarotti E and Azeredo-Oliveira MTV (1999b) Patterns of nucleolar activity during spermatogenesis of two triatomines, Panstrongylus megistus and P. herreri. Caryologia 52:177184.

Tartarotti E, Azeredo-Oliveira MTV and Ceron CR (2004) Problemática vetorial da doença de Chagas. Arq Cienc Saude 11:44-47 (Abstract in English).

Tavares MG and Azeredo-Oliveira MTV (1997) Cytogenetic studies on holocentric chromosomes of five species of triatomines (Heteroptera, Reduviidae). Cytobios 89:51-61.

Ueshima N (1979) Hemiptera II: Heteroptera. In: John, B. Animal Cytogenetics, v. 3: insecta 6. Gebrüder Borntraeger, Berlim, pp 1-117.

\section{Internet Resources}

UTHSCSA image tool for windows. Version 3. San Antonio: the University of Texas Health Science in San Antonio, c 1995/2002, http://www.uthscsa.edu. Associate Editor: Yatiyo Yonenaga-Yassuda

License information: This is an open-access article distributed under the terms of the Creative Commons Attribution License, which permits unrestricted use, distribution, and reproduction in any medium, provided the original work is properly cited. 